\title{
Comparison of Antimicrobial Efficacy of Herbal Root Canal Irrigants (Azadirachta indica, Morinda citrifolia) against Enterococcus faecalis
}

\author{
Tabassum Afshan ${ }^{1}$, Aslam Parwez ${ }^{2}$, Palagiri L Prasanna ${ }^{3}$, Meenavolu VG Bhargav ${ }^{4}$, Kurnool Balaji ${ }^{5}$, Tameem Hussain ${ }^{6}$
}

\begin{abstract}
Aim: To evaluate the antimicrobial efficacy of neem leaf extract, Morinda citrifolia and saline, against Enterococcus faecalis.

Materials and methods: A total of 120 teeth were selected for the study from the subjects between the age group of 6 and 12 years. Forty teeth were randomly assigned to one of the 3 groups corresponding to the irrigant to be tested; group I (Azadirachta indica), group II (M. citrifolia) and group III (saline). After irrigation with the respective irrigant, the post-irrigation sample was sent for the microbiological analysis to determine the antimicrobial efficacy of each irrigant.

Results: The mean E. faecalis growth of group I after irrigation was 15.48 , group II was 24.73 and group III was 40.08 . It was statistically significant ( $p$ value 0.000 ) among all the three groups. This shows that the mean E. faecalis growth was significantly higher after irrigation with saline when compared to other groups.

Conclusion: Neem leaf extract exhibited maximum inhibition against $E$. faecalis while saline exhibited the least antimicrobial efficacy with least inhibition.

Clinical significance: In the clinical setup, saline which is used as a common irrigant significantly reduces the number of bacteria in the root canal, but could not completely eliminate them. This study was an effort to find out alternative herbal irrigants which can perform effectively and are available easily for clinical practice.

Keywords: Antimicrobial efficacy, Azadirachta indica, Enterococcus faecalis, Herbal, Irrigants, Morinda citrifolia.

World Journal of Dentistry (2020): 10.5005/jp-journals-10015-1733
\end{abstract}

\section{INTRODUCTION}

Microorganisms found in the oral environment are most often the opportunistic organisms, which have the potential capability of establishing an infectious process. ${ }^{1}$ The infected root canal is seen as a habitat for more number of facultative anaerobic microorganisms. ${ }^{2}$ The main aim of an endodontic treatment is to eliminate, prevent the further entry of microorganisms from the infected root canal and provide appropriate environment for tissue healing. ${ }^{3}$

Enterococcus faecalis is one of the anaerobic gram positive bacterium generally isolated from the failed root canals and is responsible for $80-90 \%$ of enterococcal infection. It plays an essential role in persistent failure of endodontic therapy because of its ability to invade dentinal tubules, and its virulence which is attributed to its resistance to intracanal medicaments. ${ }^{4,5}$ This very nature of its to survive chemo mechanical preparation and intracanal medicaments helps it to reinfect the treated canals.

There was always an extensive search for herbal medicaments to replace the chemical irrigants in order to combat their side effects. With the substantial increase among the antibiotic resistant strains, it became inevitable to learn about herbal medicaments which possess a sound antimicrobial property to enhance the outcome of the biomechanical procedures. ${ }^{6}$ Numerous herbal extracts, having antimicrobial, anti-inflammatory, and therapeutic effects are likely to be a used as endodontic irrigants. Some of the most important herbal irrigants are as follows, neem, tulsi, aloe vera, Morinda citrifolia, Curcum longa, ${ }^{7,8}$
${ }^{1}$ Department of Pedodontics and Preventive Dentistry, Tulips Clinic, Hyderabad, Telangana, India

${ }^{2}$ Department of Pedodontics and Preventive Dentistry, Asian Multispecialty Dental Clinic, Hanamkonda, Warangal, Telangana, India ${ }^{3}$ Department of Pedodontics and Preventive Dentistry, Government Dental College and Hospital, Kadapa, Andhra Pradesh, India

${ }^{4}$ Department of Prosthodontics, Bhargavi Multispecialty Dental Clinic, Hyderabad, Telangana, India

${ }^{5}$ Department of Pedodontics and Preventive Dentistry, Family Dental Care, Hyderabad, Telangana, India

${ }^{6}$ Department of Oral and Maxillofacial Surgery, Tulips Clinic, Hyderabad, Telangana, India

Corresponding Author:Tabassum Afshan, Department of Pedodontics and Preventive Dentistry, Tulips Clinic, Hyderabad, Telangana, India, Phone: +91 9052232786, e-mail: drtabassumafshan@gmail.com

How to cite this article: Afshan T, Parwez A, Prasanna PL, et al. Comparison of Antimicrobial Efficacy of Herbal Root Canal Irrigants (Azadirachta indica, Morinda citrifolia) against Enterococcus faecalis. World J Dent 2020;11(3):206-210.

Source of support: Nil

Conflict of interest: None

Since time immemorial, Azadirachta indica commonly known as neem has been used by ancient Indian people as a cure for various diseases due to its therapeutic properties. Neem is found with active compounds like Nimbidin, Azadirachtin and Nimbinin which are responsible for its unique medicinal properties. It is one 
of the few herbal extract which has antibacterial, anticariogenic and anti-inflammatory properties along with other anti bacterial activities. $^{9-11}$

Morinda citrifolia juice (MCJ) which is commercially available as Noni, has antibacterial, antiviral, antifungal, anti-inflammatory, properties because of its compounds like L-asperuloside with alizarin making it plausible to be used as an endodontic irrigant. It is recognized to be the viable alternative for the use of sodium hypochlorite. $^{12}$

Saline has been used extensively as an irrigant to remove the debris and bacteria during the endodontic procedure. However the recommended use of physiological saline failed in complete removal of bacteria from the root canal and was ineffective as an antimicrobial agent, hence these microorganisms which cannot be washed mechanically from the root canal system, must be destroyed within, through the use of an effective antimicrobial irrigant. $^{13}$

The advantages of herbal extracts like, less side effects, cost effective, better acceptance by patients and sustainable in nature can make an effective antimicrobial irrigant in the elimination of the E. faecalis, which is one of the most commonly isolated bacterium from the failed root canals. Hence in the present study the antimicrobial efficacy of herbal irrigants; neem leaf extract, $M$. citrifolia and saline against $E$. faecalis were evaluated.

\section{Materials and Methods}

The present research was carried on subjects attending the Department of Pedodontics, CKS Theja Institute of Dental Sciences, Tirupati, Andhra Pradesh. Ethical committee clearance was obtained from the Institutional Review Board and informed written consent in full accordance with ethical principles was obtained from the parents prior to the start of the study. Healthy children of the age group 6-12 years requiring endodontic treatment, asymptomatic primary teeth with pulp necrosis, intact or less than $2 / 3$ rd roots of physiological root resorption, traumatized primary teeth with caries involving the pulp, and teeth with no mobility were included in the study.

Based on the previous studies a sample size of 40 in each group was estimated. A total of 120 teeth were selected and were analyzed by preoperative radiographs to check for pulpal and periapical status for the treatment. Each of the three groups with respective irrigant was assigned forty teeth randomly; Group I-A. indica ( $n=40)$, group II-6\% M. citrifolia juice $(n=40)$, and group III- $0.9 \%$ saline $(n=40)$. The teeth which were irrigated with saline were considered as control group.

Local anesthesia was administered and the tooth were isolated with rubber dam, local anesthesia used consisted of lignocaine with 1:80,000 adrenaline (Lignox 2\% A, Warren, Mumbai, India). A sterilized high speed air turbine with a round shaped diamond bur (BR 40: Mani, Inc., Tochigi, Japan) was used for access opening. A 10 K-file (Mani, Inc., Tochigi, Japan) was used to access the canal and the pulp tissue was extirpated from the canals using barbed broach. Bleeding was controlled with the help of cotton pellets.

\section{Preparation of Neem Leaf Extract}

Fresh neem leaves were plucked from the neem trees and washed in sterile distilled water. Twenty five grams of neem leaves and 50 $\mathrm{mL}$ of absolute alcohol were macerated for about 1-2 minutes and it was seen that the temperature was maintained at $45-50^{\circ} \mathrm{C}$. The extract prepared was passed through muslin cloth for coarse residue and the procedure was repeated. Two extracts obtained were put together and filtered through fast filter paper. On heating the mantle the alcohol part was removed from the extract and the volume was made up to $25 \mathrm{~mL}$. This output obtained was stored in screw capped containers in the refrigerator until needed.

\section{Preparation of $6 \% M$. citrifolia Juice}

$6 \mathrm{~mL}$ of $M$. citrifolia juice which is commercially available as Noni was mixed in $100 \mathrm{~mL}$ of sterile water to make it to $6 \%$ of $M$. citrifolia juice.

The herbal extract preparations were done by the researcher with the help of the staff in the pharmacy department of CKS Theja Institute of Dental Sciences, Tirupati, Andhra Pradesh.

\section{Method for Collection of Sample}

Under aseptic conditions sterile paper point was kept in the canal for 60 seconds and then shifted into a screw capped vial containing $5 \mathrm{~mL}$ of brain heart infusion broth (BHI, HIMEDIA M 210, Hi Media Laboratories Pvt Ltd, Vadhani, Mumbai) with the help of sterile tweezers. This was considered as pre-irrigation sample, and after this sample was taken the working length was calculated by using a $\mathrm{K}$ file and then confirmed by taking a radiograph. Cleaning and shaping was carried out with a stainless steel 0.02 taper K-files (Mani, Inc., Tochigi, Japan). The canal's diameter was increased up to size 35 for each canal. Ten $\mathrm{mL}$ each of the respective irrigant; $6 \% \mathrm{MC}$ (Nirogam, Trojan Pharma Ltd, HP, India), A. indica and saline (Axa Parenterals Ltd, Mumbai, India) were used during instrumentation of the canals. Five $\mathrm{mL}$ each of the three irrigants was used as a final rinse respectively on completion of the canal preparation. For all the irrigation procedures a 26 gauge sterile disposable needle with a beveled tip was used and the needle was left $1 \mathrm{~mm}$ less of the determined working length. After final irrigation with the respective irrigant the post irrigation sample was taken.

The samples collected were sent to undergo microbiological analysis to determine their individual microbiological concentration which was evaluated by colony-forming units (CFU) as seen in Figures 1 to 6 .

\section{Aerobic Culturing Technique}

The paper points obtained were immediately shifted to the sterile test tubes. Three microliters of the brain heart infusion broth were

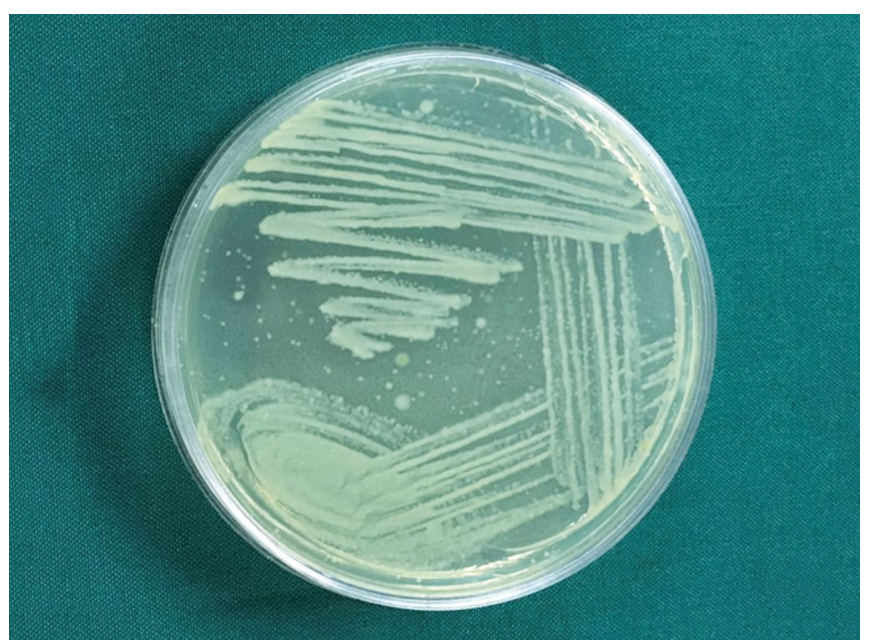

Fig. 1: Growth of E. faecalis before irrigation with neem leaf extract 


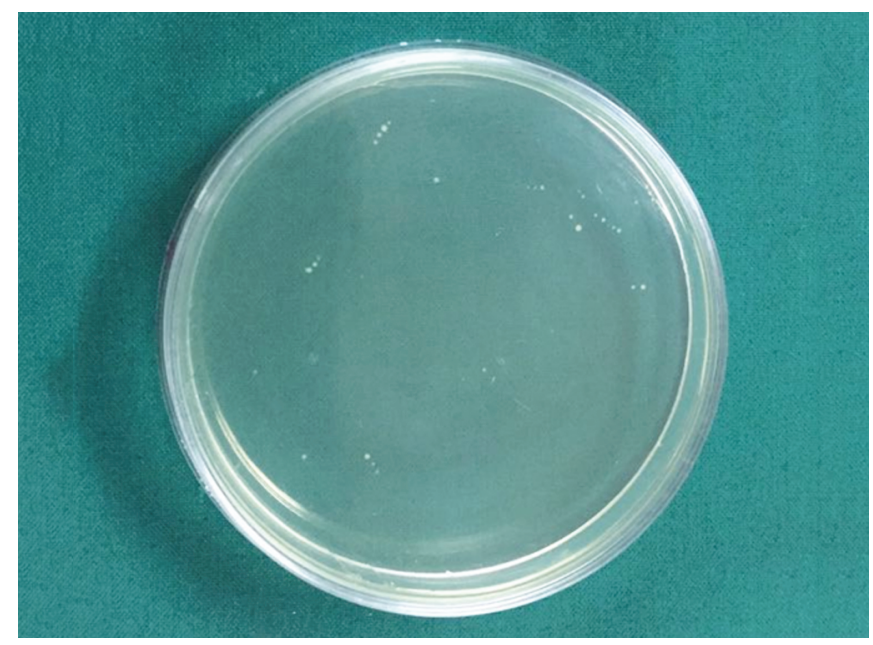

Fig. 2: Inhibition of E. faecalis after irrigation with neem leaf extract

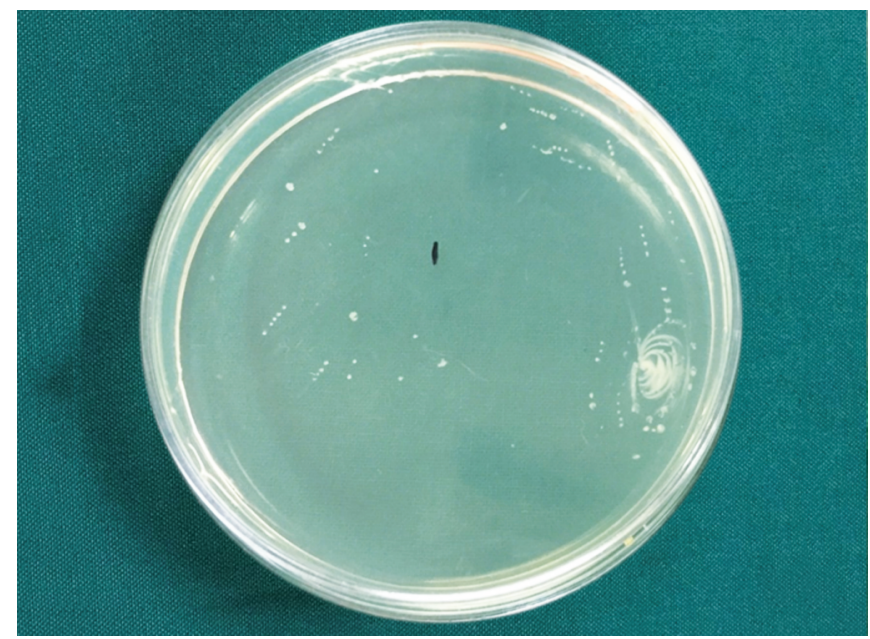

Fig. 4: Inhibition of E. faecalis after irrigation with Morinda citrifolia extract

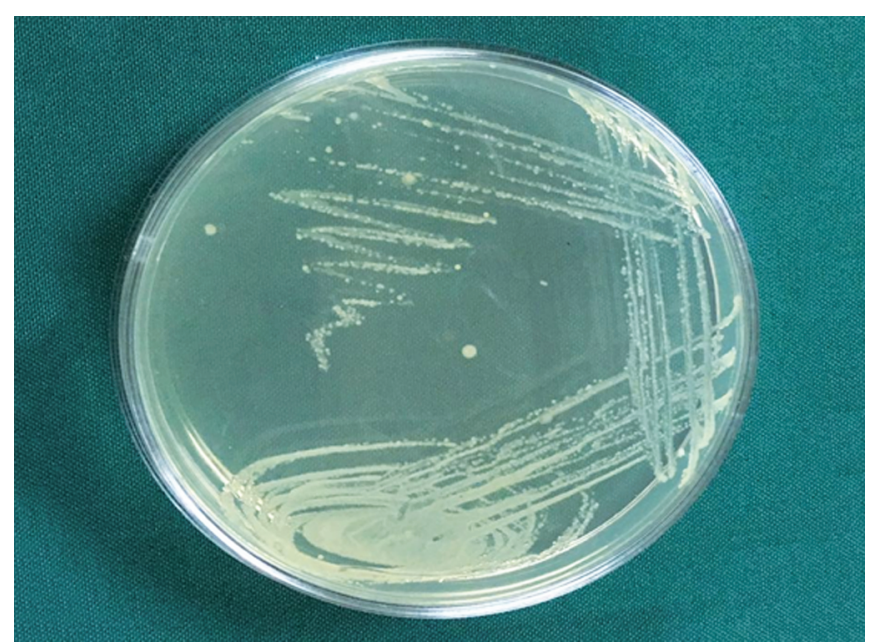

Fig. 6: Inhibition of E. faecalis after irrigation with saline

inoculated using micropipette. The inoculation loop was heated till it became red hot in the blue flame on the Bunsen burner. The streaking was done on the brain heart infusion agar plates

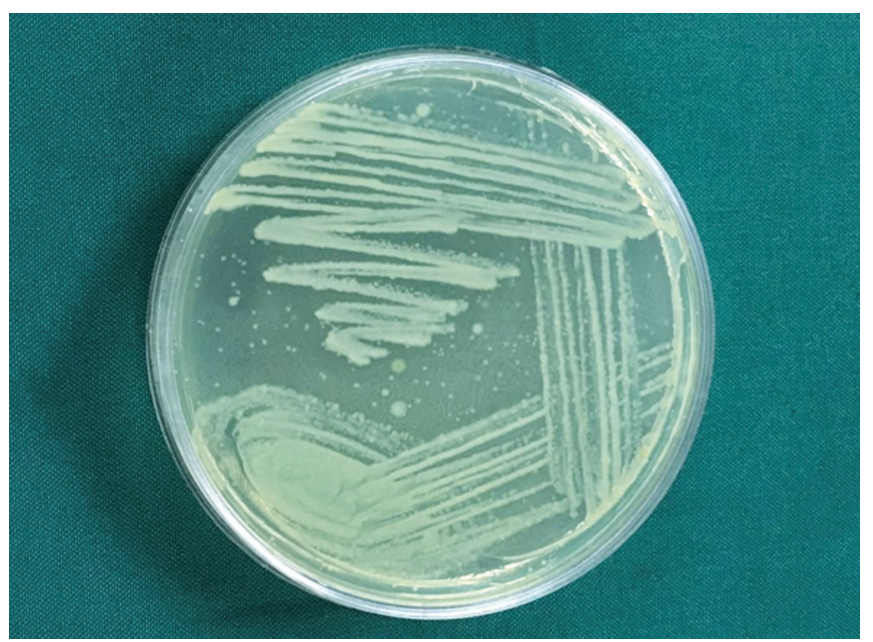

Fig. 3: Growth of E. faecalis before irrigation with Morinda citrifolia extract

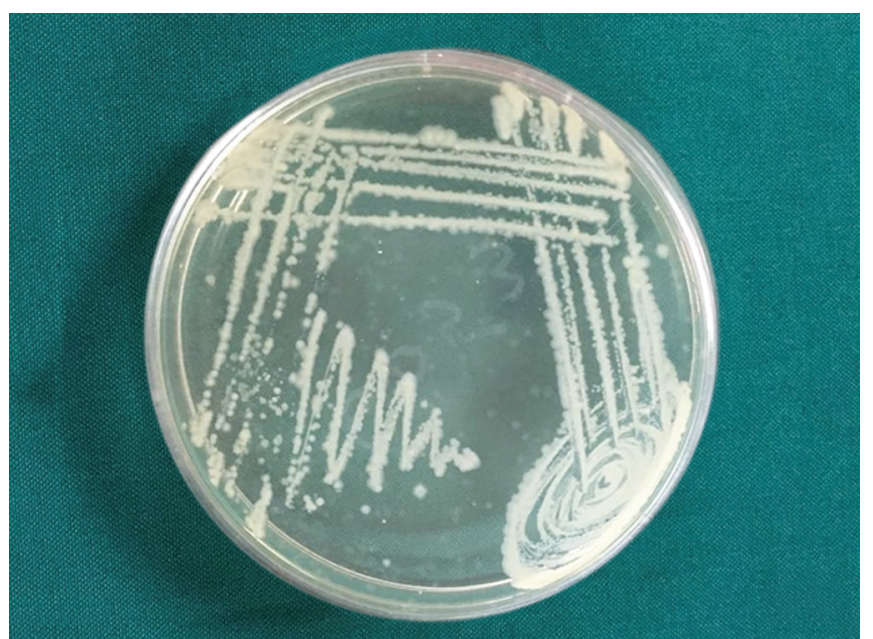

Fig. 5: Growth of E. faecalis before irrigation with saline

(HIMEDIA, M211) after the loop was cooled down at the room temperature. After that the agar plates were incubated at $37^{\circ} \mathrm{C}$ for 24 hours, and the bacterial growth was counted as CFUs using digital colony counter after 24 hours.

The E. faecalis growth as colony-forming units was collected and sent to statistical analysis using the SPSS package (version 21.0).

\section{Results}

Table 1 shows the mean $E$. faecalis growth before irrigation for group I as 44.41, group II as 44.91 and group III as 45.33. Whereasafter irrigation the mean E. faecalis growth of group I was 15.48 , group II was 24.73 and group III was 40.08 . This shows that the mean E. faecalis growth was significantly higher after irrigation with saline in group III when compared to other two groups. It was statistically significant ( $p$ value 0.000 ) among all the three groups.

Table 2 shows multiple comparisons within intra-,inter-groups before and after irrigation with post hoc tests, Tukey HSD. It shows that there was statistical insignificant difference among the three groups before irrigation. There was statistical significant difference among three groups after irrigation while comparing bothintergroup and intragroup. 


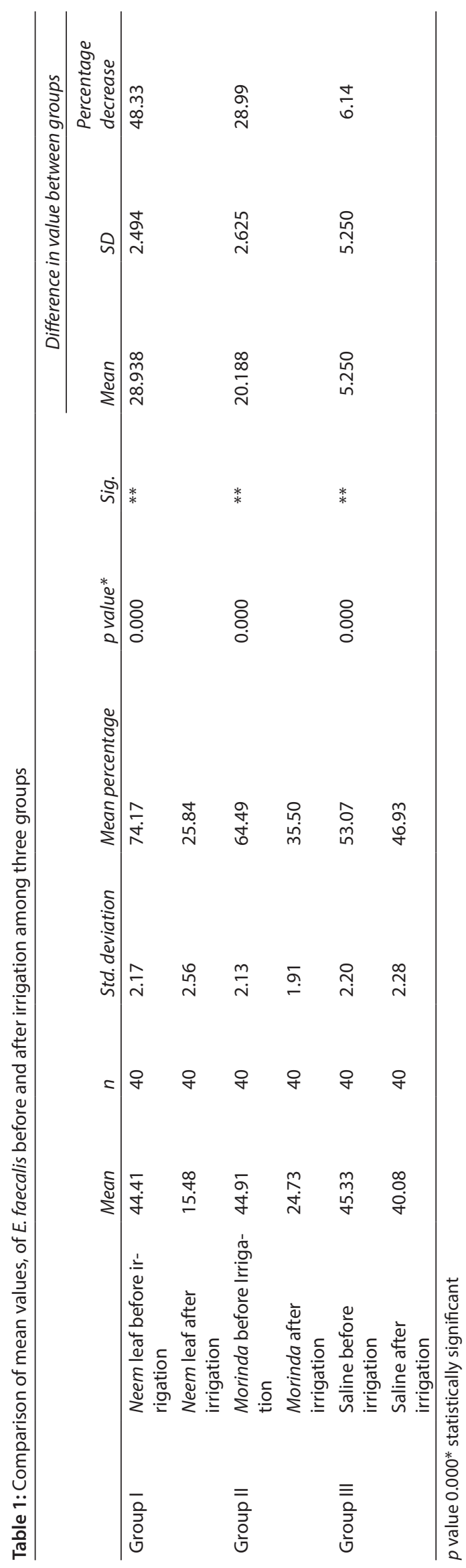

Table 2: Multiple comparisons within intra-, inter- groups before and after irrigation

\begin{tabular}{lllll}
\hline Irrigation & Group & Group & Std. error & Sig. \\
\hline Before & Group I & Group II (Morinda) & 0.484 & 0.558 \\
irrigation & (neem leaf) & Group III (saline) & 0.484 & 0.147 \\
& Group II & Group I (neem leaf) & 0.484 & 0.558 \\
& (Morinda) & Group III (saline) & 0.484 & 0.671 \\
& Group III & Group I (neem leaf) & 0.484 & 0.147 \\
After & (saline) & Group II (Morinda) & 0.484 & 0.671 \\
irrigation & Group I & Group II (Morinda) & 0.507 & $0.000^{*}$ \\
& Groem leaf) & Group III (saline) & 0.507 & $0.000^{*}$ \\
& (Morinda) & Group I (neem leaf) & 0.507 & $0.000^{*}$ \\
& Group III & Group III (saline) & 0.507 & $0.000^{*}$ \\
& (saline) & Group II (Morinda) & 0.507 & $0.000^{*}$ \\
\hline
\end{tabular}

$p$ value $0.000^{*}$ statistically significant

\section{Discussion}

The primary aim of root canal procedure is to eliminate the microbes completely from the infected root canals which allow the healing and continued maintenance of the health of the periradicular tissues. This objective is achieved mainly by cleaning and shaping along with the use of irrigants which facilitate in the removal of microorganisms. To achieve this goal several chemicals and therapeutic agents are used as irrigants. ${ }^{10}$

Root canal irrigation plays a crucial role as it helps in removal of bacteria where instrumentation is inaccessible. E. faecalis which is mostly isolated from the failed root canals can survive harsh conditions such as high alkaline environment due to biofilm formation and physicochemical properties of the organism that helps it to modify according to the prevailing environmental and nutritional conditions. ${ }^{14,15}$

Based on the results of the present study, $A$. indica has maximum inhibition ability with highest antibacterial efficacy when compared to that of M. citrifolia and saline. A study done by Podar et al. also showed similar results with the present study. ${ }^{10}$ The active compounds in A. indica like Nimbidin, Azadirachtin and Nimbinin are responsible for its antioxidant and antibacterial properties which makes it a potent substitute to sodium hypochlorite.

Chaitanya et al. in their study reported that Morinda is not as efficient as sodium hypochlorite, but better when compared to turmeric. Though this study shows that sodium hypochlorite is more effective it is not much used as irrigants in primary teeth because of necrosis that occurs in the apical region when extruded..$^{12}$ It may also be due to the fact that this study was a in vitro study where efficacy was seen only on the organisms and not in the tooth where many other factors also play a role.

However a study done by Babaji et al. observed less inhibition zone by $A$. indica when compared with sodium hypochlorite and M. citrifolia which was in contrary to the present study. This may be attributed to the fact that $E$. faecalis exhibits high prevalence in secondary endodontic infection. ${ }^{5}$

Similarly Vinothkumar et al. conducted in vitro study using real time quantitative polymerase chain reaction ( $q P C R)$. This study concluded that $A$. indica was highly efficient than sodium hypochlorite against $E$. faecalis and Candida albicans which was in accordance with the present study. ${ }^{8}$ 
Herbal irrigants are always considered safe and sound when used with efficient clinical evidence and information as they are natural. Their benefits have always made them the best replacement for the chemical substances. One of the prime examples is its easy availability and being economical. Among the wide range of benefits its minimal side effects, lack of resistance gaining nature to microbes and sustained shelf-life have made them an efficient choice as an endodontic irrigant against chemical irrigants.

Based on the results of the present study, $A$. indica has maximum inhibition ability with highest antibacterial efficacy; its biocompatible antioxidant nature with few side effects makes it an efficient endodontic irrigant. However its bitter taste which reduces the patient's ability to use the product can be neutralized by adding sweeteners and additional flavors.

As this study was an in vivo setup, direct samples from the root canals were taken for microbiological culturing. Most of the previous research was directed towards evaluating herbal irrigants in in vitro; therefore an extensive research of in vivo studies to determine their vulnerability, toxicity and capability are required.

\section{Conclusion}

A. indica was proved to be an effective alternative choice for endodontic irrigation and considered to be the best among herbal irrigants. Though M. citrifolia showed comparatively less inhibition than $A$. indica it can be used as an alternative herbal irrigant in the absence of neem leaf extract. Saline has shown the least efficacy among the three when used as an endodontic irrigant.

\section{Clinical Significance}

The results of the present study indicate that herbal extracts have potential capability to be used as an endodontic irrigant, as they are efficient in decreasing the microbial pathogens in the infected root canal. It can be considered as a beneficial replacement to sodium hypochlorite, when used in pediatric endodontics, as it does not cause any hypersensitivity or discomfort.

\section{References}

1. Siqueira Jr JF, Rocas IN, Lopes HP. Patterns of microbial colonization in primary root canal infections. Oral Surg Oral Med Oral Pathol Oral Radiol Endod 2002;93(2):174-178. DOI: 10.1067/moe.2002.119910.

2. Peters LB, Wesselink PR, Buijs JF, et al. Viable bacteria in root dentinal tubules of teeth with apical periodontitis. J Endod 2001;27(2):76-81. DOI: 10.1097/00004770-200102000-00002.

3. Mahendra M, Agrawal N, Munaga S, et al. Antimicrobial activity of different biological extracts as intracanal medicament against
Enterococcus faecalis: an in vitro study. Endodontology 2016;28(2): 166-170. DOI: 10.4103/0970-7212.195433.

4. Alagl AS, Bedi S, Almas K. Phytosolutions for Enterococcus faecalis in endodontics: an update. OHDM 2016;15(5):332-336.

5. Babaji P, Jagtap K, Lau H, et al. Comparative evaluation of antimicrobial effect of herbal root canal irrigants (Morinda citrifolia, Azadiracht aindica, Aloe vera) with sodium hypochlorite: an in vitro study. J Int Soc Prevent Communit Dent 2016;6(3):196-199. DOI: 10.4103/2231-0762.183104.

6. Prabhakar J, Senthilkumar M, Priya MS, et al. Evaluation of antimicrobial efficacy of herbal alternatives (Triphala and Green Tea Polyphenols), MTAD and 5\% sodium hypochlorite against Enterococcus faecalis biofilm formed on tooth substrate: an in vitro study. J Endod 2010;36(1):83-86. DOI: 10.1016/j.joen.2009.09.040.

7. Garg P, Tyagi SP, Sinha DJ, et al. Comparison of antimicrobial efficacy of propolis, Morinda citrifolia, Azadirachta indica, triphala, green tea polyphenols and $5.25 \%$ sodium hypochlorite against Enterococcus fecalis biofilm. Saudi Endod J 2014;4(3):122. DOI: 10.4103/16585984.138141.

8. Vinothkumar TS, Rubin MI, Balaji L, et al. In vitro evaluation of five different herbal extracts as an antimicrobial endodontic irrigant using real time quantitative polymerase chain reaction. J Conserv Dent 2013;16(2):167-170. DOI: 10.4103/0972-0707.108208.

9. Lakshmi T, Krishnan V, Rajendran R, et al. Azadirachta indica: a herbal panacea in dentistry - an update. Pharmacogn Rev 2015;9(17):41-44. DOI: 10.4103/0973-7847.156337.

10. Podar R, Kulkarni GP, Dadu SS, et al. In vivo antimicrobial efficacy of $6 \%$ Morinda citrifolia, Azadirachta indica, and 3\% sodium hypochlorite as root canal irrigants. Eur J Dent 2015;9(4):529-534. DOI: 10.4103/13057456.172615.

11. Divia AR, Nair MG, Varughese JM, et al. A comparative evaluation of Morinda citrifolia, green tea polyphenols, and Triphala with 5\% sodium hypochlorite as an endodontic irrigant against Enterococcus faecalis: an in vitro study. Dent Res J (Isfahan) 2018;15(2):117-122. DOI: 10.4103/1735-3327.226526.

12. Chaitanya BV, Somisetty KV, Diwan A. Comparison of antibacterial efficacy of turmeric extract, Morinda citrifolia and 3\% sodium hypochlorite on Enterococcus faecalis: an in vitro study. J Clin Diagn Res 2016;10(10):ZC55-ZC57. DOI: 10.7860/JCDR/2016/19718.8650.

13. Ohara $P$, Torabinejad M, Kettering JD. Antibacterial effect of various endodontic irrigants on selected anaerobic bacteria. Endod Dent Traumatol 1993;9(3):95-100.DOI: 10.1111/j.1600-9657.1993.tb00258.x.

14. Pandey S, Shekhar R, Paul R, et al. A comparative evaluation and effectiveness of different antimicrobial herbal extracts as endodontic irrigants against Enterococcus faecalis and Candida albicans - an in vitro study. Univ J Dent Sci 2018;4(2):75-81.

15. Karkare SR, Ahire NP, Khedkar SU. Comparative evaluation of antimicrobial activity of hydro alcoholic extract of Aloe vera, garlic, and $5 \%$ sodium hypochlorite as root canal irrigants against Enterococcus faecalis: an in vitro study. J Indian Soc Pedod Prev Dent 2015;33(4):274-278. DOI: 10.4103/0970-4388.165658. 